\title{
RANDOM AND FREE POSITIVE MAPS WITH APPLICATIONS TO ENTANGLEMENT DETECTION
}

\author{
BENOÎT COLLINS, PATRICK HAYDEN, AND ION NECHITA
}

\begin{abstract}
We apply random matrix and free probability techniques to the study of linear maps of interest in quantum information theory. Random quantum channels have already been widely investigated with spectacular success. Here, we are interested in more general maps, asking only for $k$-positivity instead of the complete positivity required of quantum channels. Unlike the theory of completely positive maps, the theory of $k$-positive maps is far from being completely understood, and our techniques give many new parametrized families of such maps.

We also establish a conceptual link with free probability theory, and show that our constructions can be obtained to some extent without random techniques in the setup of free products of von Neumann algebras.

Finally, we study the properties of our examples and show that for some parameters, they are indecomposable. In particular, they can be used to detect the presence of entanglement missed by the partial transposition test, that is, PPT entanglement. As an application, we considerably refine our understanding of PPT states in the case where one of the spaces is large whereas the other one remains small.
\end{abstract}

\section{CONTEnTs}

1. Introduction

2. Positive maps: notations and facts 3

3. A model via free probability 4

3.1. Freeness and free products 4

3.2. Maps associated to probability measures 5

3.3. Examples 5

3.4. Quantum states detected by "free" maps $\quad 7$

4. A finite dimensional, random version $\quad 9$

4.1. Norm behaviour of products of random matrices $r$

4.2. The finite dimensional, random matrix model $\quad 10$

5. GUE quantum states and maps 11

5.1. PPT entanglement 12

5.2. Random separability 13

5.3. Indecomposability 15

$\begin{array}{ll}\text { References } & 15\end{array}$

\section{INTRODUCTION}

Completely positive maps play a privileged role in quantum information theory because they provide the quantum mechanical generalization of the notion of a noisy channel. The Stinespring theorem provides a useful classification of these maps that also justifies their physical relevance as ignorance-induced noisy summaries of the unitary evolution on a larger system [30]. Random methods have played an important role in the study of noisy quantum channels, notably in the proofs

Date: April 10, 2018. 
of capacity theorems $[27,21,17]$ and in the construction of channels with unusual properties [18, $28,14]$, including channels violating the additivity conjecture [19, 16, 12, 6].

Maps with weaker positivity properties are of interest in quantum information theory precisely because they fail in subtle ways to be physically realizable. Most importantly, positive but not completely positive maps acting on entangled states may fail to preserve positivity, mapping states to non-positive operators. Since such maps will always preserve positivity for separable quantum states, which are by definition only classically correlated, positive but not completely positive maps can be used to detect the presence of entanglement. The most famous such test is partial transposition; the states with no entanglement detectable this way are known as the PPT states [25]. In this article, we use free probability and random matrix techniques to construct new families of positive but not completely positive maps and apply these maps to the study of entanglement.

As an illustration of the power of the method, we consider quantum states of a bipartite space of finite dimension. Motivated originally by debates about whether room temperature nuclear magnetic resonance experiments were capable of producing entanglement, researchers determined some time ago that there is a neighborhood of separable states in the vicinity of the maximally mixed state [9], and have produced successively sharper estimates on the size of that neighborhood [15]. We consider the related question of perturbing the maximally mixed state by adding to it a random Hermitian traceless matrix. This question has been studied in turn by Aubrun, Szarek, Ye, and others $[2,3,4]$. In the limit where at least one of the systems has large dimension, one finds sharp transitions between separable states, PPT states and, finally, states whose entanglement can be detected using partial transposition. We construct new positive maps capable of detecting entanglement to which the partial transposition test is insensitive.

Formally, let $\mathcal{A}, \mathcal{B}$ be two $C^{*}$-algebras. Positive elements in a $C^{*}$ algebra are elements $x$ that are self-adjoint and that can be written as $x=y y^{*}$ for some $y$ in the $C^{*}$-algebra. A positive linear map $\Phi: \mathcal{A} \rightarrow \mathcal{B}$ is a map that sends a positive element to a positive element. It is well-known that if $\Phi$ is positive, then $\Phi_{k}=\mathrm{id}_{k} \otimes \Phi: \mathcal{M}_{k}(\mathbb{C}) \otimes \mathcal{A} \rightarrow \mathcal{M}_{k}(\mathbb{C}) \otimes \mathcal{B}$ is not necessarily positive, unless $\mathcal{A}$ or $\mathcal{B}$ are commutative. For example if $\mathcal{A}=\mathcal{B}=\mathcal{M}_{n}(\mathbb{C}), n \geq 2$ and $\Phi$ is the transpose map, then, $\Phi_{k}$ is not positive as soon as $k \geq 2$.

However if $\Phi_{k}$ is positive, then for $l \leq k$, the map $\Phi_{l}$ is clearly positive too. A map $\Phi$ such that $\Phi_{k}$ is positive is said to be $k$-positive. A completely positive map is one that has this property for all integers $k$. Note that if $\mathcal{A}=\mathcal{M}_{n}(\mathbb{C})$ and $\mathcal{B}=\mathcal{M}_{d}(\mathbb{C}), \min (n, d)$-positive is equivalent to completely positive [29, Corollary 4.19]. There are many examples of maps that are $k$ positive but not $(k+1)$-positive, but the classification of positive, or even $k$-positive maps is far from complete.

One of the aims of this paper is to provide a new systematic method to obtain $k$-positive maps from $\mathcal{M}_{n}(\mathbb{C}) \rightarrow \mathcal{M}_{d}(\mathbb{C})$, based on free probability techniques. More precisely, for all integers $n, k$ such that $n \geq k$, we describe infinite families of maps $\Phi: \mathcal{M}_{n}(\mathbb{C}) \rightarrow \mathcal{M}_{d}(\mathbb{C})$ that are $k$-positive but not $(k+1)$-positive. Our families are defined for any $d$ large enough (and the threshold depends on $n, k)$, and their positivity properties depend on the behaviour of the support of the free convolution powers of a given compactly supported probability measure.

Our first main result is Theorem 3.2, and can be stated as follows (we refer to Section 3 for details):

Theorem 1.1. In a non-commutative probability space where a copy of the $n \times n$ matrices $\mathcal{M}_{n}(\mathbb{C})$ is free from a self-adjoint element $X$ of distribution $\mu$, consider the map $\Phi_{\mu}: \mathcal{M}_{n}(\mathbb{C}) \rightarrow \mathcal{M}$ given by $\Phi_{\mu}\left(E_{i j}\right)=E_{1 i} X E_{j 1}$, with $E=\left(E_{i j}\right)_{i, j \in\{1, \ldots, n\}}$ the basis of matrix units. Then, the map $\Phi_{\mu}$ is $k$-positive if and only if $\operatorname{supp}\left(\mu^{\boxplus n / k}\right) \subseteq[0, \infty)$.

The second main series of results looks into a random matrix approximation of the above theorem, and focuses on the Gaussian setup. We summarize the results below, and refer to Section 5 for details. 
Theorem 1.2. Let $Z_{d}$ be a shifted $G U E$ random matrix in $\mathcal{M}_{n}(\mathbb{C}) \otimes \mathcal{M}_{d}(\mathbb{C})$ of mean 2 and variance $\alpha \in[0,1)$. Then there is a linear map $\Phi_{d}: \mathcal{M}_{n}(\mathbb{C}) \rightarrow \mathcal{M}_{n}(\mathbb{C})$ such that its Choi matrix is again a shifted GUE matrix (which depends on $Z_{d}$ ), of mean $(2+\varepsilon) / \sqrt{n}$ and variance 1 . With probability one as $d \rightarrow \infty$ :

(1) The matrix $Z_{d}$ is positive and PPT.

(2) The map $\Phi_{d}$ is positive. However, $\Phi_{d}$ is not completely positive, provided that $2+\varepsilon<\sqrt{n}$.

(3) As soon as $2(2+\varepsilon)<\alpha \sqrt{n}$, the map $\Phi_{d}$ detects the entanglement present in $Z_{d}$.

Our paper is organized as follows. Section 2 sets up some notation about positive and $k$-positive maps, and recalls some results about their Choi matrices. Section 3 is about free probability: it recalls abstract facts about freeness, the free additive convolution semigroup, and it gives our main new construction of $k$-positive maps. Section 4 is about the finite dimensional random approximation of the model of Section 3, and Section 5 specifies to the case of non-centered GUE random matrices, with applications to typical radii of convergence for PPT vs. separability, along with non-decomposability results for our random maps.

Acknowledgements. B.C.'s research was partly supported by NSERC, ERA, and Kakenhi funding. P.H.'s research is supported by CIFAR, FQXi and the Simons Foundation. I.N.'s research has been supported by a von Humboldt fellowship and by the ANR projects OSQPI 2011 BS01 00801 and RMTQIT ANR-12-IS01-0001-01. Both B.C. and I.N. were supported by the ANR project STOQ ANR-14-CE25-0003.

\section{Positive maps: notations And faCts}

We denote by $\mathcal{M}_{n}(\mathbb{C})$ the algebra of $n \times n$ complex matrices. Let $E=\left(E_{i j}\right)_{i, j \in\{1, \ldots n\}}$ be the basis of matrix units, i.e. $E_{i j} E_{k l}=E_{i l} \delta_{j k}$ and $E_{i j}^{*}=E_{j i}$. For a $C^{*}$-algebra $\mathcal{A}$, let $\Phi: \mathcal{M}_{n}(\mathbb{C}) \rightarrow \mathcal{A}$ be a linear map. Its Choi matrix in the basis $E$, denoted by $C_{\Phi}$ is an element of $\mathcal{M}_{n}(\mathbb{C}) \otimes \mathcal{A}$ defined as

$$
C_{\Phi}=\sum_{i, j=1}^{n} E_{i j} \otimes \Phi\left(E_{i j}\right) .
$$

Denoting by $B_{d} \in \mathbb{C}^{d} \otimes \mathbb{C}^{d}$ the normalized Bell state

$$
B_{d}=\frac{1}{\sqrt{d}} \sum_{i=1}^{d} e_{i} \otimes e_{i},
$$

the Choi matrix can also be written as

$$
C_{\Phi}=d\left[\mathrm{id}_{d} \otimes \Phi\right]\left(B_{d} B_{d}^{*}\right) .
$$

A map $\Phi: \mathcal{M}_{n}(\mathbb{C}) \rightarrow \mathcal{A}$ is called $k$-positive if the dilated application

$$
\Phi \otimes \mathrm{id}_{k}: \mathcal{M}_{n}(\mathbb{C}) \otimes \mathcal{M}_{k}(\mathbb{C}) \rightarrow \mathcal{A} \otimes \mathcal{M}_{k}(\mathbb{C})
$$

is positive. Moreover, such a map is called completely positive if it is $k$-positive for all $k$. According to a celebrated result of Choi [13], $\Phi$ is completely positive if and only if $C_{\Phi}$ is positive in $\mathcal{M}_{n}(\mathbb{C}) \otimes \mathcal{A}$. A characterization of $k$-positivity using the Choi-Jamiołkowski isomorphism has been obtained in $[26]$.

We recall the following result from [22], characterizing $k$-positivity of maps.

Proposition 2.1. [22, Proposition 2.2] Consider a linear map $\Phi: \mathcal{M}_{n}(\mathbb{C}) \rightarrow \mathcal{B}(\mathcal{H})$, where $\mathcal{H}$ is a Hilbert space. The following are equivalent:

(1) The map $\Phi$ is k-positive.

(2) For all vectors $x=\sum_{i=1}^{k} y_{i} \otimes z_{i}$, with $y_{i} \otimes z_{i} \in \mathbb{C}^{n} \otimes \mathcal{H},\left\langle x, C_{\Phi} x\right\rangle \geq 0$. 
(3) The operator $\left(I_{n} \otimes P\right) C_{\Phi}\left(I_{n} \otimes P\right)$ is positive semidefinite for any rank $k$ orthogonal projection $P \in \mathcal{B}(\mathcal{H})$.

(4) The operator $\left(P \otimes 1_{\mathcal{H}}\right) C_{\Phi}\left(P \otimes 1_{\mathcal{H}}\right)$ is positive semidefinite for any rank $k$ orthogonal projection $P \in \mathcal{M}_{n}(\mathbb{C})\left(1_{\mathcal{H}}\right.$ is the identity operator in $\left.\mathcal{B}(\mathcal{H})\right)$.

\section{A MOdel VIA FREe PROBABILITy}

We start this section by recalling some facts from free probability theory that will be used throughout the paper. We then introduce our construction of linear maps from probability measures and we characterize their block-positivity properties (random matrix versions of these results will be considered in Section 4). Finally, we discuss some simple, but illuminating examples in the final subsection.

3.1. Freeness and free products. The monographs [33] and [24] are excellent introductions to free probability theory; here, we recall only the concepts needed in this paper.

A *-non-commutative probability space is a unital $*$-algebra $\mathcal{A}$ endowed with a tracial state $\phi$, i.e. a linear map $\phi: \mathcal{A} \rightarrow \mathbb{C}$ satisfying $\phi(a b)=\phi(b a), \phi\left(a a^{*}\right) \geq 0, \phi(1)=1$. An element of $\mathcal{A}$ is called a non-commutative random variable.

Let $\mathcal{A}_{1}, \ldots, \mathcal{A}_{k}$ be subalgebras of $\mathcal{A}$ having the same unit as $\mathcal{A}$. They are said to be free if for all $a_{i} \in \mathcal{A}_{j_{i}}(i=1, \ldots, k)$ such that $\phi\left(a_{i}\right)=0$, one has

$$
\phi\left(a_{1} \cdots a_{k}\right)=0
$$

as soon as $j_{1} \neq j_{2}, j_{2} \neq j_{3}, \ldots, j_{k-1} \neq j_{k}$. Collections $S_{1}, S_{2}, \ldots$ of random variables are said to be free if the unital subalgebras they generate are free.

Let $\left(a_{1}, \ldots, a_{k}\right)$ be a $k$-tuple of self-adjoint random variables and let $\mathbb{C}\left\langle X_{1}, \ldots, X_{k}\right\rangle$ be the free *-algebra of non-commutative polynomials on $\mathbb{C}$ generated by the $k$ self-adjoint indeterminates $X_{1}, \ldots, X_{k}$.

The joint distribution of the family $\left\{a_{i}\right\}_{i=1}^{k}$ is the linear form

$$
\begin{aligned}
\mu_{\left(a_{1}, \ldots, a_{k}\right)}: \mathbb{C}\left\langle X_{1}, \ldots, X_{k}\right\rangle & \rightarrow \mathbb{C} \\
P & \mapsto \phi\left(P\left(a_{1}, \ldots, a_{k}\right)\right) .
\end{aligned}
$$

Given a $k$-tuple $\left(a_{1}, \ldots, a_{k}\right)$ of free random variables such that the distribution of $a_{i}$ is $\mu_{a_{i}}$, the joint distribution $\mu_{\left(a_{1}, \ldots, a_{k}\right)}$ is uniquely determined by the $\mu_{a_{i}}$ 's.

In particular, $\mu_{a_{1}+a_{2}}$ and $\mu_{a_{1} a_{2}}$ depend only on $\mu_{a_{1}}$ and $\mu_{a_{2}}$. The notations $\mu_{a_{1}+a_{2}}=\mu_{a_{1}} \boxplus \mu_{a_{2}}$ and $\mu_{a_{1} a_{2}}=\mu_{a_{1}} \nabla \mu_{a_{2}}$ were introduced in Voiculescu's works [31, 32]; the operations $\boxplus$ and $\nabla$ are called the free additive, respectively free multiplicative convolution. Moreover, for any compactly supported probability measure $\mu$, one can define its free additive convolution powers $\mu^{\boxplus T}$ for any real parameter $T \geq 1$, see [24, Corollary 14.13].

The following result will be quite useful for us and was proved by Nica and Speicher [24, Exercise 14.21].

Lemma 3.1. Let $a, p$ be free elements in a non-commutative probability space $(\mathcal{A}, \phi)$ and assume that $p$ is a self-adjoint projection of rank $t \in(0,1)$ and that a is a self-adjoint random variable having distribution $\mu$. Then, the distribution of $t^{-1}$ pap in $(p \mathcal{A} p, \phi(p \cdot))$ is $\mu^{\boxplus 1 / t}$.

Finally, we discuss free products. Given $\left(\mathcal{A}_{i}, \phi_{i}\right)_{i \in I}$ a family of non-commutative probability spaces, there exists a non-commutative probability space $(\mathcal{A}, \phi)$ in which all $\left(\mathcal{A}_{i}, \phi_{i}\right)$ can be embedded in a trace-preserving way such that they are free with respect to each other. This is called Voiculescu's free product construction, and we will need it below. There exists a $*$-algebra version of this construction, a $C^{*}$-algebra and a von Neumann algebra construction. If $\mathcal{A}$ is generated by the $\mathcal{A}_{i}$ 's, we write $(\mathcal{A}, \phi)=*_{i \in I}\left(\mathcal{A}_{i}, \phi_{i}\right)$. 
3.2. Maps associated to probability measures. We now introduce the main idea of this paper, a construction of a linear map depending on a probability measure $\mu$. We start with a compactly supported probability measure $\mu$ on $\mathbb{R}$ and fix an integer $n$. We will be interested in the space $L^{\infty}(\mathbb{R}, \mu)$. Note that this space is spanned as a von Neumann algebra by the operator $x \mapsto x$, which we will denote by $X$. By construction, $X$ is a self-adjoint operator and its spectrum is the support of $\mu$.

We consider the von Neumann probability space free product [33]

$$
(\tilde{\mathcal{M}}, \operatorname{tr} * \mathbb{E}):=\left(\mathcal{M}_{n}(\mathbb{C}), \operatorname{tr}\right) *\left(L^{\infty}(\mathbb{R}, \mu), \mathbb{E}\right)
$$

and the contracted von Neumann probability space $(\mathcal{M}, \tau)$ where $\mathcal{M}=E_{11} \tilde{\mathcal{M}} E_{11}$ is the contracted algebra of $\tilde{\mathcal{M}}$ and $\tau$ is the restriction of $\operatorname{tr} * \mathbb{E}$ appropriately normalized (by a factor $n$ ).

We consider the map $\Phi_{\mu}: \mathcal{M}_{n}(\mathbb{C}) \rightarrow \mathcal{M}$ given by

$$
\Phi_{\mu}\left(E_{i j}\right)=E_{1 i} X E_{j 1} .
$$

Our main result is as follows:

Theorem 3.2. Let $\mu$ be a compactly supported probability measure. The map $\Phi_{\mu}$ defined above is $k$-positive if and only if $\operatorname{supp}\left(\mu^{\boxplus n / k}\right) \subseteq[0, \infty)$.

Proof. The map $\Phi_{\mu}$ was constructed in such a way that its Choi matrix has distribution $\mu$. Indeed, the von Neumann algebra $\mathcal{M}$ is a corner of $\tilde{\mathcal{M}}$, so the map $a \mapsto \sum_{i, j=1}^{n} E_{i j} \otimes E_{1 i} a E_{j 1}$ implements an isomorphism between $\tilde{\mathcal{M}}$ and $\mathcal{M}_{n}(\mathcal{M})$; hence, $C_{\Phi_{\mu}}=X$. For any rank $k$ orthogonal projection $P \in \mathcal{M}_{n}(\mathbb{C})$, the distribution of the element $\left(P \otimes 1_{\mathcal{M}}\right) C_{\Phi_{\mu}}\left(P \otimes 1_{\mathcal{M}}\right)$ is $\left[(1-k / n) \delta_{0}+k / n \delta_{1}\right] \otimes \mu$. By the Nica-Speicher result 3.1 above, this distribution has positive support if and only if $\operatorname{supp}\left(\mu^{\boxplus n / k}\right) \subseteq$ $[0, \infty)$. The result follows now from an application of Proposition 2.1.

The remainder of the paper is devoted to the study of the maps $\Phi_{\mu}$ and to applications of the above construction to Quantum Information Theory.

3.3. Examples. Let us start the subsection devoted to examples by making some general purpose remarks. First, note that in order for the condition in Theorem 3.2 to hold for some value of $k$, the measure $\mu$ must have non-negative average. Indeed, the average of $\mu^{\boxplus n / k}$ is $n / k$ times the average of $\mu$. It follows that, if one starts with a probability distribution $\mu$ having negative mean, the measures $\mu^{\boxplus n / k}$ cannot have support in $[0, \infty)$. In particular, in order to obtain non-trivial examples, we have to rule out any distributions which are symmetric with respect to the origin.

Similarly, a measure $\mu$ whose support is already in $\mathbb{R}_{+}$will not be interesting for us because the corresponding map $\Phi_{\mu}$ will automatically be completely positive and thus $k$-positive for all $1 \leq k \leq n$ (recall that we are ultimately interested in positive maps which are not completely positive). In the following, we are interested in non-trivial examples of measures $\mu$, i.e. measures such that

$$
\operatorname{supp}(\mu) \nsubseteq[0, \infty) \text { but } \operatorname{supp}\left(\mu^{\boxplus T}\right) \subseteq[0, \infty) \text { for some } T \geq 1 .
$$

3.3.1. The semicircular case. We start with semicircular distributions, which will play a central role in Section 5. As mentioned above, in order to produce non-trivial examples of maps $\Phi_{\mu}$, one should rule out symmetric distributions $\mu$; we consider next shifted (non-centered) semicircle distributions. Let us consider $\mathrm{SC}_{a, \sigma}$ the Wigner semicircle distribution of mean $a$ and variance $\sigma^{2}$ :

$$
\mathrm{SC}_{a, \sigma}=\frac{\sqrt{4 \sigma^{2}-(x-a)^{2}}}{2 \pi \sigma^{2}} \mathbf{1}_{[a-2 \sigma, a+2 \sigma]}(x) d x .
$$

Note that the support of the semicircle measure is $[a-2 \sigma, a+2 \sigma]$. The semicircle distribution plays a role in free probability theory analogous to the role played by the Gaussian distribution in classical probability. Its free cumulants are all zero, except for the first two, which read $\kappa_{1}=a$, $\kappa_{2}=\sigma^{2}$. Since the free cumulants linearize the free additive convolution [24, Proposition 12.3], 
their values for $\mathrm{SC}_{a, \sigma}^{\boxplus n / k}$ are $\kappa_{1}=a n / k$, respectively $\kappa_{2}=\sigma^{2} n / k$, all the others being zero. Hence, $\mathrm{SC}_{a, \sigma}^{\boxplus n / k}=\mathrm{SC}_{a n / k, \sigma \sqrt{n / k}}$, and thus the support of $\mathrm{SC}_{a, \sigma}^{\boxplus n / k}$ is

$$
\operatorname{supp}\left(\mathrm{SC}_{a, \sigma}^{\boxplus n / k}\right)=\left[\frac{a n}{k}-2 \sigma \sqrt{\frac{n}{k}}, \frac{a n}{k}+2 \sigma \sqrt{\frac{n}{k}}\right]
$$

In particular, we have:

Proposition 3.3. Let $n$ be an integer, $a \in \mathbb{R}$, and $\sigma>0$, and consider the map $\Phi_{\mathrm{SC}}^{(a, \sigma)}: \mathcal{M}_{n}(\mathbb{C}) \rightarrow$ $\mathcal{M}$ associated to the semicircle distribution $\mathrm{SC}_{a, \sigma}$. The map $\Phi_{\mathrm{SC}}^{(a, \sigma)}$ is $k$-positive if and only if $k \leq a^{2} n /\left(4 \sigma^{2}\right)$. In particular, for any $n$ and any $1 \leq k<n$, there exist parameters $a, \sigma>0$ such that the above map is $k$-positive but not $k+1$-positive.

3.3.2. The Marchenko-Pastur case. Here we consider $m_{a, t}$ to be the distribution of the random variable $1-a X_{t}$ where $X_{t}$ has a Marcenko-Pastur (or free Poisson of parameter $t$ ) distribution and $a>0$. We recall that the free Poisson law of parameter $t$ is given by (see, e.g. [24, Definition $12.12])$ :

$$
\pi_{t}=\max (1-t, 0) \delta_{0}+\frac{\sqrt{4 t-(x-1-t)^{2}}}{2 \pi x} 1_{[1+t-2 \sqrt{t}, 1+t+2 \sqrt{t}]} d x,
$$

and that it has the semigroup property $\pi_{s} \boxplus \pi_{t}=\pi_{s+t}$ (this follows from the fact that the free cumulants of $\pi_{t}$ read $\kappa_{p}=t, \forall p \geq 1$ ). In particular, this implies for our purposes that the bottom of the spectrum of $m_{a, t}$ is located at $1-a(1+t+2 \sqrt{t})$. It follows from Nica and Speicher's Lemma 3.1 that if $p$ is a free projection of rank $k / n$, the bottom of the spectrum of the operator $p\left(1-a X_{t}\right) p$ is located at

$$
1-\frac{k}{n} a\left(1+t \frac{n}{k}+2 \sqrt{t n / k}\right) .
$$

We summarize what we obtained above as follows.

Proposition 3.4. Let $n$ be an integer, $a, t>0, \Phi_{\mathrm{MP}}^{(t)}: \mathcal{M}_{n}(\mathbb{C}) \rightarrow \mathcal{M}$ whose Choi map is associated to the non-commutative random variable $1-a X_{t}$. If $1-\frac{k}{n} a\left(1+t \frac{n}{k}+2 \sqrt{t n / k}\right) \geq 0$ but $1-\frac{k+1}{n} a(1+$ $\left.t \frac{n}{k+1}+2 \sqrt{t n /(k+1)}\right)<0$ then the map $\Phi_{\mathrm{MP}}^{(t)}$ is $k$-positive but not $(k+1)$-positive. Moreover, for each $n, k$ there exists a choice of a,t that satisfies the above inequalities.

3.3.3. The 'small rank projection' case. Now we are interested in the case where $X=1-a P$ where $P$ is a projection of rank $\varepsilon$ small (i.e. $\tau(P)=\varepsilon \in(0,1)$ ).

Proposition 3.5. As $\varepsilon \rightarrow 0$, the threshold in a to obtain $k$-positivity converges to $n / k$.

Proof. Let us consider some fixed $\varepsilon>0$. Then, there exists a variable $Y$ having a Marchenko-Pastur distribution of parameter $\varepsilon$ such that, for any $\eta \geq 6 \sqrt{\varepsilon}$,

$$
1-(a+\eta) Y \leq X \leq 1-(a-\eta) Y .
$$

Indeed, recall that the Marchenko-Pastur distribution of parameter $\varepsilon<1$ can be written as

$$
\pi_{\varepsilon}=(1-\varepsilon) \delta_{0}+\varepsilon \tilde{\pi}_{\varepsilon},
$$

where $\tilde{\pi}_{\varepsilon}$ is a probability measure supported on the compact interval $\left[(1-\sqrt{\varepsilon})^{2},(1+\sqrt{\varepsilon})^{2}\right]$. The result follows after applying Proposition 3.4 and letting first $\varepsilon \rightarrow 0$ and then $\eta \rightarrow 0$. 
3.4. Quantum states detected by "free" maps. Let $\mathcal{D}_{n}$ be the set of $n$-dimensional quantum states

$$
\mathcal{D}_{n}:=\left\{\rho \in \mathcal{M}_{n}: \rho \geq 0 \text { and } \operatorname{Tr} \rho=1\right\},
$$

and consider the set of separable states

$$
\mathcal{S E P}_{n, m}=\left\{\sum_{i} t_{i} \rho_{i} \otimes \sigma_{i}: t_{i} \geq 0, \rho_{i} \in \mathcal{D}_{n}, \sigma_{i} \in \mathcal{D}_{m}, \sum_{i} t_{i}=1\right\} \subseteq \mathcal{D}_{n m} .
$$

Given a positive map $\Phi: \mathcal{M}_{n} \rightarrow \mathcal{M}_{d}$, we introduce the convex body of $n \cdot m$-dimensional quantum states which remain positive under the partial action of $\Phi$

$$
K_{\Phi, m}=\left\{\rho \in \mathcal{D}_{n m}:\left[\Phi \otimes \mathrm{id}_{m}\right](\rho) \geq 0\right\} .
$$

We first record the following elementary result:

Lemma 3.6. For any positive map $\Phi$ and any dimension $m$, the set $K_{\Phi, m}$ is a convex body and it contains the set $\mathcal{S E P}_{n, m}$ of separable states. If $\Phi$ is not $m$-positive, then $K_{\Phi, m}$ is a strict subset of $\mathcal{D}_{n m}$.

Let us turn our attention to the particular case of a map $\Phi_{\mu}$ as defined in the previous sections. We start with the following proposition, showing that any non-trivial $\Phi_{\mu}$ detects pure entangled states.

Proposition 3.7. Let $\mu$ be a compactly supported probability measure such that $\operatorname{supp}\left(\mu^{\boxplus n}\right) \subseteq[0, \infty)$, but $\operatorname{supp}\left(\mu^{\boxplus n / k}\right) \cap(-\infty, 0) \neq \emptyset$, for all $2 \leq k \leq n$. Then, the set $K_{\Phi_{\mu}, m}$ contains all separable states but no pure entangled state. Put differently, $\Phi_{\mu}$ detects perfectly the entanglement of pure states.

Proof. The claim about separable states follows from the fact that the map $\Phi_{\mu}$ is (1-)positive. Let $p$ be a rank one self-adjoint projection on $\mathcal{M}_{n}(\mathbb{C}) \otimes \mathcal{M}_{k}(\mathbb{C})$ that is not separable (i.e. $p$ is a multiple of an entangled state). Let $q \in \mathcal{M}_{n}(\mathbb{C})$ be the orthogonal projection on the range of the partial trace of $p$; since $p$ is entangled, $\operatorname{rk}(q) \geq 2$.

By direct inspection, studying the positivity of $\left[\Phi_{\mu} \otimes \mathrm{id}_{m}\right](p)$ is exactly the same problem as studying the positivity of $\left(q \otimes 1_{\mathcal{M}}\right) X\left(q \otimes 1_{\mathcal{M}}\right)$; indeed, using a Schur map argument, one can assume without loss of generality that the eigenvalues of the partial trace of $p$ are all equal. However, by our assumptions, the latter element does not have a positive spectrum. Therefore, $K_{\Phi_{\mu}, m}$ does not contain pure entangled states.

Let us now introduce two intermediate sets between the set of separable states and the set of all quantum states, corresponding to the reduction criterion $[10,20]$ and the positive partial transposition (or PPT) criterion [25]:

$$
\begin{aligned}
& \mathcal{R E} \mathcal{D}_{n, m}=\left\{\rho \in \mathcal{D}_{n m}: I_{n} \otimes\left[\operatorname{Tr}_{n} \otimes \mathrm{id}_{m}\right](\rho)-\rho \geq 0\right\} \\
& \mathcal{P} \mathcal{P} \mathcal{T}_{n, m}=\left\{\rho \in \mathcal{D}_{n m}:\left[\top_{n} \otimes \operatorname{id}_{m}\right](\rho) \geq 0\right\},
\end{aligned}
$$

where $T$ denotes the transposition map. It is known [10] that these sets satisfy the following chain of inclusions (which all become strict for large enough values of $n, m$ )

$$
\mathcal{S E P}_{n, m} \subseteq \mathcal{P} \mathcal{P} \mathcal{T}_{n, m} \subseteq \mathcal{R} \mathcal{E} \mathcal{D}_{n, m} \subseteq \mathcal{D}_{n m}
$$

We show next that the maps corresponding to the "small rank projection" measures (see Section 3.3.3) detect the same entangled states as the reduction criterion, in a suitable asymptotic regime.

Theorem 3.8. In the setup of subsection 3.3.3, with parameters $a=n, \varepsilon \rightarrow 0$, the convex body $K_{\Phi, m}$ tends to $\mathcal{R} \mathcal{E} \mathcal{D}_{n, m}$.

Before we prove this result, let us provide an equivalent, but more general form of the reduction criterion. 
Lemma 3.9. Let $\Phi: \mathcal{M}_{n}(\mathbb{C}) \rightarrow \mathcal{A}$ be a (non-unital) embedding of $*$-algebras, sending the matrix units $E_{i j}$ of $\mathcal{M}_{n}(\mathbb{C})$ to elements $F_{i j} \in \mathcal{A}$. Then, for any integer $m$ and for any quantum state $\rho=\sum_{i j=1}^{n} E_{i j} \otimes \rho_{i j} \in \mathcal{D}_{n m}$,

$$
I_{n} \otimes\left[\operatorname{Tr}_{n} \otimes \operatorname{id}_{m}\right](\rho)-\rho \geq 0 \Longleftrightarrow 1_{\mathcal{A}} \otimes \sum_{i=1}^{n} \rho_{i i}-\sum_{i, j=1}^{n} F_{i j} \otimes \rho_{i j} \geq 0 .
$$

Proof of Theorem 3.8. As in the context of section 3.3.3 we are looking at a Choi matrix of the form $X_{\varepsilon}=1-n P$ where $P$ is a projection of trace $\varepsilon$. Let $\Phi_{\varepsilon}$ be the corresponding map (obtained from $X_{\varepsilon}$ via Theorem 3.2).

For a given $\mathcal{D}_{n m} \ni \rho=\sum_{i j=1}^{n} E_{i j} \otimes \rho_{i j} \in \mathcal{D}_{n m}$, we have

$$
\left[\Phi_{\varepsilon} \otimes \mathrm{id}_{m}\right](\rho)=1_{\mathcal{M}} \otimes \sum_{i=1}^{n} \rho_{i i}-\sum_{i, j=1}^{n} F_{i j}^{(\varepsilon)} \otimes \rho_{i j} \geq 0,
$$

where $F_{i j}^{(\varepsilon)}=n E_{1 i} P E_{j 1}$. Let us show now that the $F_{i j}^{(\varepsilon)}$ are approximate matrix units, i.e. they verify the $*$-algebra relations for matrix units up to some vanishing precision as $\varepsilon \rightarrow 0$. To show that, as $\varepsilon \rightarrow 0$, for all $i, j, k, l$,

$$
\left\|F_{i j}^{(\varepsilon)} F_{k l}^{(\varepsilon)}-\delta_{j k} F_{i l}^{(\varepsilon)}\right\| \rightarrow 0
$$

we just have to compute

$$
n^{2} E_{1 i} P E_{j 1} E_{1 k} P E_{l 1}-n \delta_{j k} E_{1 i} P E_{l 1}=n E_{1 i}\left(n P E_{j k} P-\delta_{j k} P\right) E_{l 1} .
$$

We show next that, as $\varepsilon \rightarrow 0$,

$$
\left\|n P E_{j k} P-\delta_{j k} P\right\| \rightarrow 0 .
$$

Let us first consider the case $j=k$. Again from Nica and Speicher's Lemma 3.1, it follows that the distribution of the element $P\left(n E_{j j}-I\right) P$ in the reduced non-commutative probability space $P \mathcal{M} P$ is that of the dilation of the free additive power $D_{\varepsilon}\left[\left(n^{-1} \delta_{n-1}+\left(1-n^{-1}\right) \delta_{-1}\right)^{\boxplus 1 / \varepsilon}\right]$. Let $\varepsilon=1 / N$, for some (large) integer $N$. Then, by the super-convergence result of Bercovici and Voiculescu [7, Theorem 7] (see also [23, Example 1]), we have that the support of

$$
D_{N^{-1 / 2}}\left[\left(n^{-1} \delta_{n-1}+\left(1-n^{-1}\right) \delta_{-1}\right)^{\boxplus N}\right]
$$

is contained, for $N$ large enough, into some fixed compact interval. The normalization in $N^{-1}$ shows the desired convergence in norm to zero. The case $j \neq k$ is treated in a similar manner, by considering the symmetrized version $\left\|P\left(E_{j k}+E_{k j}\right) P\right\|=2\left\|P E_{j k} P\right\|$.

By multiplying on the left and on the right equation (3) by $E_{1 i}$, resp. $E_{l 1}$ which are both of operator norm one, one obtains (2); note that in all the computations above, $n$ is a fixed parameter.

It follows from [8, Proposition II.8.3.22] that approximate matrix units can be approximated by true (exact) matrix units: there exist true matrix units $G_{i j}^{(\varepsilon)} \in \mathcal{M}$ such that, for all $i, j, \| F_{i j}^{(\varepsilon)}-$ $G_{i j}^{(\varepsilon)} \| \rightarrow 0$ as $\varepsilon \rightarrow 0$. Define

$$
\Psi_{\varepsilon, m}(\rho)=1_{\mathcal{M}} \otimes \sum_{i=1}^{n} \rho_{i i}-\sum_{i, j=1}^{n} G_{i j}^{(\varepsilon)} \otimes \rho_{i j} \geq 0 .
$$

By the previous lemma, $\rho \in \mathcal{R E D}_{n, m}$ iff. $\Psi_{\varepsilon, m}(\rho) \geq 0$ in $\mathcal{M}$. But we have

$$
\left\|\left[\Phi_{\varepsilon} \otimes \mathrm{id}_{m}\right](\rho)-\Psi_{\varepsilon, m}(\rho)\right\|=\left\|\sum_{i, j=1}^{n}\left(F_{i j}^{(\varepsilon)}-G_{i j}^{(\varepsilon)}\right) \otimes \rho_{i j}\right\| \leq n^{2} \sup _{i, j}\left\|F_{i j}^{(\varepsilon)}-G_{i j}^{(\varepsilon)}\right\| \rightarrow 0,
$$

finishing the proof. 
In other words, an infinitesimally small free projection captures exactly the set of quantum states whose entanglement is detected by the reduction criterion. Since $\mathcal{R} \mathcal{E} \mathcal{D}_{n, m} \supseteq \mathcal{P} \mathcal{P} \mathcal{T}_{n, m}$, an important question would is to check whether the sets $K_{\Phi_{\mu}, m}$ can be compared to $\mathcal{P} \mathcal{P} \mathcal{T}_{n, m}$ for appropriate measures $\mu$. Although we do not address this question directly, we will see with Theorem 5.4 that with random techniques, a result of this flavor holds.

We finish this section by a general open question. For all $n, k, l$, we introduce the set

$$
K_{n, k, m}^{\text {free }}:=\bigcap_{\mu: \operatorname{supp}\left(\mu^{\boxplus n / k}\right) \subset[0, \infty)} K_{\Phi_{\mu}, m} .
$$

We call it the collection of freely detectable quantum states in $\mathcal{D}_{n m}$.

In view of the preliminary results of this section, we believe that this set is of some interest in quantum information theory. The sequel of this paper will show that suitable approximations of this set have a very strong entanglement detection power. This leads us to stating the following question, that has to our mind an interest both in free probability and quantum information theory:

Question 3.10. Can one give a description of $K_{n, k, m}^{\text {free }}$ ? We know this set contains the collection k-separable states (Theorem 3.2), but how much bigger is it? Are there values of the parameters $n, m, m$ for which the set $K_{n, k, m}^{\text {free }}$ is precisely the set of $k$-separable states from $\mathcal{D}_{n m}$ ?

\section{A Finite Dimensional, RANDOM VERSiON}

Theorem 3.2 and the construction of the maps $\Phi_{\mu}$ give a way to construct new families of positive but not completely positive maps. The results in Section 3 are not very concrete in the sense that the compression of free products of von Neumann algebras is a rather abstract object. In this section, we show that it is possible to interpret the previous results as the limiting case of random maps between finite dimensional matrix algebras. In particular, we obtain a large class of linear maps between matrix algebras that are $k$ positive but not $k+1$ positive. For this we need a result about norm approximations.

4.1. Norm behaviour of products of random matrices. We recall that if $X$ is a $d$-dimensional self-adjoint matrix, its empirical eigenvalue distribution is $d^{-1} \sum_{i=1}^{d} \delta_{\lambda_{i}}$, where $\lambda_{i}$ are the eigenvalues of $X$. For any probability measure $\mu$ on the real line, its repartition function is defined as $F_{\mu}: t \mapsto$ $\mu((-\infty, t])$.

For the purposes of this section, we will say that a sequence of repartition functions $f_{d}$ converges to a repartition function $f$ if for all $\varepsilon>0$, there exists an $d_{0}$ such that for all $d \geq d_{0}$,

$$
\forall t \in \mathbb{R}, \quad f(t-\varepsilon)-\varepsilon \leq f_{d}(t) \leq f(t+\varepsilon)+\varepsilon .
$$

Theorem 4.1. Let $A_{d}, B_{d}$ be independent positive self-adjoint random matrices in $\mathcal{M}_{d}(\mathbb{C})$, such that at least one of $A_{d}$ or $B_{d}$ has a distribution invariant under unitary conjugation. Let $f_{d}$ be the repartition function of $A_{d}$ and $g_{d}$ be the repartition function of $B_{d}$. Assume that the (a priori random) repartition functions $f_{d}, g_{d}$ converge almost surely respectively to $f, g$ which are repartition functions of two self-adjoint, bounded and freely independent random variables $x$ and $y$. Assume also that the operator norm of $A_{d}$ (resp. $B_{d}$ ) converges to the operator norm of $x$ (resp. $y$ ). Then, almost surely as $d \rightarrow \infty$,

$$
\left\|A_{d} B_{d}\right\| \rightarrow\|x y\| .
$$

The above result is a special case of a much more general class of results, see $[11,5]$. 
4.2. The finite dimensional, random matrix model. In this section, we fix again a compactly supported probability measure $\mu$ and an integer $n$. For each integer $d$, we introduce a real-valued diagonal matrix $X_{d} \in \mathcal{M}_{n}(\mathbb{C}) \otimes \mathcal{M}_{d}(\mathbb{C})$ whose empirical eigenvalue distribution converges to $\mu$ and whose extremal eigenvalues converge to the respective extrema of the support of $\mu$ :

$$
\begin{aligned}
& \lim _{d \rightarrow \infty} \lambda_{\min }\left(X_{d}\right)=\min \{x: x \in \operatorname{supp}(\mu)\} \\
& \lim _{d \rightarrow \infty} \lambda_{\max }\left(X_{d}\right)=\max \{x: x \in \operatorname{supp}(\mu)\} .
\end{aligned}
$$

Let also $U_{d}$ be a random unitary matrix in $\mathcal{U}_{n d}$ distributed according to the Haar measure on the unitary group. We introduce the map $\Phi_{\mu, d}: \mathcal{M}_{n}(\mathbb{C}) \rightarrow \mathcal{M}_{d}(\mathbb{C})$ whose Choi matrix is the random matrix $C_{d}:=U_{d} X_{d} U_{d}^{*} \in \mathcal{M}_{n}(\mathbb{C}) \otimes \mathcal{M}_{d}(\mathbb{C})$; this can be done using the hermiticity preserving map $\leftrightarrow$ self-adjoint matrix incarnation of the Choi-Jamiołkowski isomorphism. More precisely, we have

$$
\forall X \in \mathcal{M}_{n}(\mathbb{C}), \quad \Phi_{\mu, d}(X)=[\operatorname{Tr} \otimes \mathrm{id}]\left(C_{d} \cdot X^{\top} \otimes I_{d}\right) .
$$

We can now state the main result of this section.

Theorem 4.2. The sequence of random linear maps $\left(\Phi_{\mu, d}\right)_{d}$ has the following properties:

(1) If $\operatorname{supp}\left(\mu^{\boxplus n / k}\right) \subset(0, \infty)$, then, almost surely as $d \rightarrow \infty, \Phi_{\mu, d}$ is $k$-positive.

(2) If $\operatorname{supp}\left(\mu^{\boxplus n / k}\right) \cap(-\infty, 0) \neq \emptyset$, then, almost surely as $d \rightarrow \infty, \Phi_{\mu, d}$ is not $k$-positive.

Proof. Let $C_{d}$ be the Choi matrix of the map $\Phi_{\mu, d}$, i.e. $C_{d}=U_{d} X_{d} U_{d}^{*}$ for a sequence of real diagonal matrices $\left(X_{d}\right)_{d}$ converging in distribution to $\mu$. The assumption implies that there exists some positive constant $\delta>0$ such that $\operatorname{supp}\left(\mu^{\boxplus n / k}\right) \subset[\delta, \infty)$. Fix $M>\max \operatorname{supp}(\mu)$ and let $\varepsilon>0$ be a small constant which will be fixed later. Fix an $\varepsilon$-net $\mathcal{Q}$ of projections of rank $k$ on $\mathcal{M}_{n}(\mathbb{C})$ (for the operator norm). This net can be chosen to be finite because the collection of projections is a compact space. In other words, for any rank $k$ projection $P \in \mathcal{M}_{n}(\mathbb{C})$, there exists a rank $k$ projection $Q \in \mathcal{Q}$ such that $\|P-Q\| \leq \varepsilon$.

For a fixed element $Q \in \mathcal{Q}$, we obtain, using Theorem 4.1 and Lemma 3.1, almost surely as $d \rightarrow \infty$

$$
\lim _{d \rightarrow \infty} \lambda_{\min }\left[\left.\left(Q \otimes I_{d} \cdot C_{d} \cdot Q \otimes I_{d}\right)\right|_{\operatorname{ran}(Q) \otimes \mathbb{C}^{d}}\right]=\frac{k}{n} \min \operatorname{supp}\left(\mu^{\boxplus n / k}\right) \geq \frac{k \delta}{n} .
$$

Taking the intersection of a finite number of almost sure events, we obtain the same conclusion for all elements $Q$ of $\mathcal{Q}$. Moreover, since, for all rank $k$ projections $P$, we have almost surely

$$
\left\|P \otimes I_{d} \cdot C_{d} \cdot P \otimes I_{d}-Q \otimes I_{d} \cdot C_{d} \cdot Q \otimes I_{d}\right\| \leq\|P-Q\| \cdot\left\|C_{d}\right\| \leq M \varepsilon,
$$

we conclude that, almost surely as $d \rightarrow \infty$,

$$
\lim _{d \rightarrow \infty} \min _{\text {projection of rank } k} \lambda_{\min }\left[\left.\left(P \otimes I_{d} \cdot C_{d} \cdot P \otimes I_{d}\right)\right|_{\operatorname{ran}(P) \otimes \mathbb{C}^{d}}\right] \geq \frac{k \delta}{n}-M \varepsilon,
$$

quantity which is positive as soon as $\varepsilon<(k \delta) /(n M)$. By Proposition 2.1, this completes the first part.

To prove the second point, consider any fixed orthogonal projection $P \in \mathcal{M}_{n}(\mathbb{C})$ of rank $k$. Using Theorem 4.1, we obtain that, almost surely as $d \rightarrow \infty$, the smallest non-trivial eigenvalue of the matrix $\left(P \otimes I_{d}\right) C_{d}\left(P \otimes I_{d}\right)$ converges to $k / n$ times the bottom of the support of $\mu^{\boxplus n / k}$, which is a negative constant. Therefore, by Proposition 2.1, the map $\Phi_{\mu, d}$ is not positive, almost surely as $d \rightarrow \infty$.

When comparing the result above with Theorem 3.2, the reader will notice that one needs a gap around zero in the random matrix formulation. This is due to the fact that one needs to avoid eigenvalues fluctuating around zero in order to draw a conclusion about the positivity of a matrix. Finally, from the above theorem, one can immediately deduce the following corollary, simply by replacing the almost sure notion of convergence of random variables with the weaker one of convergence in probability. 
Corollary 4.3. The sequence of random linear maps $\left(\Phi_{\mu, d}\right)_{d}$ has the following properties:

(1) If $\operatorname{supp}\left(\mu^{\boxplus n / k}\right) \subset(0, \infty)$, then

$$
\lim _{d \rightarrow \infty} \mathbb{P}\left[\Phi_{\mu, d} \text { is } k \text {-positive }\right]=1 .
$$

(2) If $\operatorname{supp}\left(\mu^{\boxplus n / k}\right) \cap(-\infty, 0) \neq \emptyset$, then

$$
\lim _{d \rightarrow \infty} \mathbb{P}\left[\Phi_{\mu, d} \text { is k-positive }\right]=0 .
$$

\section{GUE QUANTUM STATES AND MAPS}

In this section, we focus on (possibly traceless) random matrices from the Gaussian Unitary Ensemble (GUE). The second chapter of the monograph [1] is an excellent exposition of the subject. The interest of GUE random matrices is two-fold. Firstly, it is the case where the norm behavior of random matrices is best understood. Secondly, GUE matrices are both unitarily invariant (their distribution is invariant with respect to unitary conjugations) and Wigner matrices (the entries of the random matrix are independent and identically distributed, up to symmetries). We write $\mathrm{i}=\sqrt{-1}$ in order to avoid confusions with the index $i$ of matrix elements.

Definition 5.1. A random matrix $Z \in \mathcal{M}_{d}(\mathbb{C})$ is said to have the $\mathrm{GUE}_{d}$ distribution if its entries are as follows:

$$
Z_{i j}= \begin{cases}X_{i i} / \sqrt{d}, & \text { if } i=j \\ \left(X_{i j}+\mathrm{i} Y_{i j}\right) / \sqrt{2 d}, & \text { if } i<j \\ \bar{Z}_{j i}, & \text { if } i>j,\end{cases}
$$

where $\left\{X_{i j}, Y_{i j}\right\}_{i, j=1}^{d}$ are i.i.d. centered, standard real Gaussian random variables.

We recall next the Wigner theorem, as well as the stronger, norm convergence of GUE matrices.

Theorem 5.2. Consider a sequence of $\mathrm{GUE}_{d}$ random matrices $X_{d} \in \mathcal{M}_{d}(\mathbb{C})$. Then, almost surely as $d \rightarrow \infty$, the empirical distribution of $X_{d}$ converges weakly to a standard semicircular distribution:

$$
\frac{1}{d} \sum_{i=1}^{d} \lambda_{i}\left(X_{d}\right) \rightarrow \mathrm{SC}_{0,1}
$$

Moreover, the norm of $X_{d}$ converges almost surely to the maximum of the support:

$$
\lim _{d \rightarrow \infty}\left\|X_{d}\right\|=2 \text {. }
$$

We start with the following simple but important lemma, which will allow us to construct different realizations of GUE matrices acting on a tensor product space. Below, that the matrices $\left\{E_{i j}\right\}$ denote the matrix units of $\mathcal{M}_{n}(\mathbb{C})$.

Lemma 5.3. For any integers $n, d$, consider a family of i.i.d. $\mathrm{GUE}_{d}$ random matrices $\left\{X_{i j}, Y_{i j}\right\}_{i, j=1}^{n}$. Define

$$
\begin{aligned}
\mathcal{M}_{n d}(\mathbb{C}) \ni Z:= & \frac{1}{\sqrt{n}} \sum_{i=1}^{n} E_{i i} \otimes X_{i i} \\
& +\frac{1}{\sqrt{2 n}} \sum_{1 \leq i<j \leq n} E_{i j} \otimes\left(X_{i j}+\mathrm{i} Y_{i j}\right) \\
& +\frac{1}{\sqrt{2 n}} \sum_{1 \leq i<j \leq n} E_{j i} \otimes\left(X_{i j}-\mathrm{i} Y_{i j}\right) .
\end{aligned}
$$

Then, $Z$ has a $\mathrm{GUE}_{n d}$ distribution. 
Proof. The $(n d)^{2}$ elements of $Z$ are centered, Gaussian, independent (up to symmetries) random variables. The normalizations are chosen in such a way that the variances are exactly those of a $\mathrm{GUE}_{n d}$ random matrix.

5.1. PPT entanglement. We are now going to construct an asymptotically positive semidefinite and PPT matrix $Z_{d} \in \mathcal{M}_{n}(\mathbb{C}) \otimes \mathcal{M}_{d}(\mathbb{C})$ as well as a map $\Phi_{d}$ which will detect the entanglement in $Z_{d}$. Both constructions are inspired from Lemma 5.3.

Consider a fixed integer $n \geq 1$ and fixed parameters $\varepsilon>0$ and $\alpha \in(0,1)$. Let $\left\{X_{i j}\right\}_{1 \leq i \leq j \leq n}$ and $\left\{Y_{i j}\right\}_{1 \leq i<j \leq n}$ be two families of i.i.d. GUE ${ }_{d}$ random matrices. Define the matrices

$$
\begin{aligned}
\mathcal{M}_{n d}(\mathbb{C}) \ni Z_{d}:=2 I_{n} \otimes I_{d}-\alpha & {\left[\frac{1}{\sqrt{n}} \sum_{i=1}^{n} E_{i i} \otimes X_{i i}\right.} \\
& +\frac{1}{\sqrt{2 n}} \sum_{1 \leq i<j \leq n} E_{i j} \otimes\left(X_{i j}+\mathrm{i} Y_{i j}\right) \\
& \left.+\frac{1}{\sqrt{2 n}} \sum_{1 \leq i<j \leq n} E_{j i} \otimes\left(X_{i j}-\mathrm{i} Y_{i j}\right)\right],
\end{aligned}
$$

and

$$
\begin{aligned}
\mathcal{M}_{n d}(\mathbb{C}) \ni C_{d}:=\frac{2+\varepsilon}{\sqrt{n}} I_{n} \otimes I_{d} & +\frac{1}{\sqrt{n}} \sum_{i=1}^{n} E_{i i} \otimes X_{i i}^{\top} \\
& +\frac{1}{\sqrt{2 n}} \sum_{1 \leq i<j \leq n} E_{i j} \otimes\left(X_{i j}^{\top}-\mathrm{i} Y_{i j}^{\top}\right) \\
& +\frac{1}{\sqrt{2 n}} \sum_{1 \leq i<j \leq n} E_{j i} \otimes\left(X_{i j}^{\top}+\mathrm{i} Y_{i j}^{\top}\right),
\end{aligned}
$$

and consider the linear map $\Phi_{d}: \mathcal{M}_{n}(\mathbb{C}) \rightarrow \mathcal{M}_{d}(\mathbb{C})$ whose Choi matrix is $C_{d}$. In other words, for an input $\rho \in M_{n}(\mathbb{C})$,

$$
\Phi_{d}(\rho)=\sum_{i, j=1}^{n} \rho_{i j} C_{d}(i, j),
$$

where $C_{d}(i, j) \in \mathcal{M}_{d}(\mathbb{C})$ denotes the $(i, j)$ block of $C_{d}$.

Theorem 5.4. For the linear maps defined in (4),(5), and (6), the following statements hold:

(1) For all values of $n, \alpha$, almost surely as $d \rightarrow \infty$, the random matrix $Z_{d}$ is positive semidefinite and PPT.

(2) For all values of $n, \varepsilon$, almost surely as $d \rightarrow \infty$, the linear map $\Phi_{d}$ is positive.

(3) If $B_{d}$ denotes the normalized Bell state (1), then, almost surely as $d \rightarrow \infty$,

$$
\lim _{d \rightarrow \infty}\left\langle B_{d},\left[\Phi_{d} \otimes \operatorname{id}_{d}\right]\left(Z_{d}\right) B_{d}\right\rangle=2(2+\varepsilon) \sqrt{n}-\alpha n .
$$

In particular, for any choice of parameters such that $2(2+\varepsilon)<\alpha \sqrt{n}$, the matrix $Z_{d}$ is, almost surely as $d \rightarrow \infty$, asymptotically PPT and entangled.

Proof. For the first point, note that the matrix $Z_{d}$ can be written as $Z_{d}=2 I_{n d}-\alpha \tilde{Z}_{d}$, where $\tilde{Z}_{d}$ is a $\mathrm{GUE}_{n d}$ matrix (see Lemma 5.3. It follows that the support of its asymptotic spectral distribution is the interval $[2-2 \alpha, 2+2 \alpha]$, which is supported on the positive half-line; strong convergence of $Z_{d}$ proves that $Z_{d}$ is asymptotically positive semidefinite. It is also PPT, since transposed GUE matrices are still GUE. 
For the second claim, to show positivity of the map $\Phi_{d}$ having $C_{d}$ as a Choi matrix, we are going to use Theorem 4.2. Indeed, at fixed $n$, as $d \rightarrow \infty$, the random matrix $C_{d}$ has asymptotic spectral distribution $\mu=\mathrm{SC}_{(2+\varepsilon) / \sqrt{n}, 1}$. We get $\mu^{\boxplus n}=\mathrm{SC}_{(2+\varepsilon) \sqrt{n}, \sqrt{n}}$, which has support $[\varepsilon \sqrt{n},(4+\varepsilon) \sqrt{n}] \subset$ $(0, \infty)$, hence $\Phi_{d}$ is asymptotically positive, by Theorem 4.2 .

Let us now show that the third point holds. Denoting by $A(i, j) \in \mathcal{M}_{d}(\mathbb{C})$ the $(i, j)$ block of a matrix $A \in \mathcal{M}_{n d}(\mathbb{C})$, a direct linear algebra computation shows that

$$
\begin{aligned}
\left\langle B_{d},\left[\Phi_{d} \otimes \mathrm{id}_{d}\right]\left(Z_{d}\right) B_{d}\right\rangle= & \frac{1}{d} \sum_{i, j=1}^{n} \operatorname{Tr}\left[Z_{d}(i, j) C_{d}(i, j)^{\top}\right] \\
=\frac{1}{d} & {\left[\frac{2(2+\varepsilon)}{\sqrt{n}} n d-\frac{\alpha}{n} \sum_{i=1}^{n} \operatorname{Tr}\left(X_{i i}^{2}\right)\right.} \\
& \left.\quad-\frac{\alpha}{n} \sum_{1 \leq i<j \leq n} \operatorname{Tr}\left[\left(X_{i j}+\mathrm{i} Y_{i j}\right)\left(X_{i j}-\mathrm{i} Y_{i j}\right)\right]\right]
\end{aligned}
$$

Using, for all $i, j$, almost surely as $d \rightarrow \infty$

$$
\lim _{d \rightarrow \infty} \frac{1}{d} \operatorname{Tr}\left(X_{i j}^{2}\right)=\lim _{d \rightarrow \infty} \frac{1}{d} \operatorname{Tr}\left(Y_{i j}^{2}\right)=1
$$

and

$$
\lim _{d \rightarrow \infty} \frac{1}{d} \operatorname{Tr}\left(X_{i j} Y_{i j}\right)=\lim _{d \rightarrow \infty} \frac{1}{d} \operatorname{Tr}\left(X_{i j}\right)=\lim _{d \rightarrow \infty} \frac{1}{d} \operatorname{Tr}\left(Y_{i j}\right)=0
$$

we can conclude.

The construction of the matrix $Z_{d}$ can be interpreted as follows. Let us replace the notation $Z_{d}$ by $Z_{d}(\alpha)$, i.e. view the Gaussian component of $Z_{d}$ as a 'direction' and $\alpha$ as an intensity when choosing at random from the vicinity of the maximally mixed state (everything up to trivial scalars). With probability 1 we have that as $d \rightarrow \infty, Z_{d}(1)$ is a multiple of a PPT state, and that actually it is on the boundary of PPT in the sense that if $\alpha=1$ is replaced by a greater value of $\alpha$, it will leave the convex cone of PPT matrices.

The following corollary is itself of interest:

Corollary 5.5. For any $n>16$, there exist $\alpha, \varepsilon$ such that the map $\Phi_{d}$ detects the entanglement in the PPT matrix $Z_{d}$.

In other words, as soon as $n>16$, the maps we introduced in sections 3 and 4 can detect PPT states that are not separable. The analysis above also shows that the maps $\Phi_{d}$ become very efficient as entanglement detection tools for $n$ large. Indeed,

Corollary 5.6. For any $1>\alpha>4 / \sqrt{n}$ the positive matrix $Z_{d}$ is PPT entangled.

This following result is a direct extension of Theorem 5.4.

Proposition 5.7. Fix parameters $n, l$ such that $n>16 l$. For any $1>\alpha>4 \sqrt{l} / \sqrt{n}$, the positive matrix $Z_{d}$ from (4) is PPT but not l-separable, with high probability as $d \rightarrow \infty$.

Proof. The proof goes exactly as for Theorem 5.4, except that one has to replace positivity by $l$-positivity, which affects the threshold accordingly.

5.2. Random separability. Finally, we would like to finish this section by mentioning that the threshold $4 / \sqrt{n}$ from Corollary 5.6, although perhaps not optimal, is of the right order with respect to $n$, when $n$ is large. 
Theorem 5.8. Let $X_{d}$ be a standard GUE random matrix in $\mathcal{M}_{n d}(\mathbb{C})$, and $\alpha \in(0,1)$ be a fixed constant. With probability 1 as $d \rightarrow \infty$, the matrix $2 I_{n d}+\alpha X_{d}$ is separable as soon as $\frac{2}{\alpha}>2+\frac{4(n-1)}{\sqrt{n}}$. In particular, if $\alpha<\sqrt{n} /(2(n-1)+\sqrt{n})$, the hermitian matrix $Z_{d}$ defined in (4) is, almost surely as $d \rightarrow \infty$, separable.

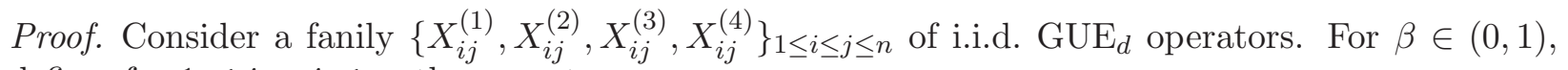
define, for $1 \leq i<j \leq n$, the operators

$$
\begin{aligned}
& T_{i j}^{(1)}=\left(E_{i i}+E_{i j}+E_{j i}+E_{j j}\right) \otimes\left(\beta X_{i j}^{(1)}+2 I_{d}\right) \\
& T_{i j}^{(2)}=\left(E_{i i}-E_{i j}-E_{j i}+E_{j j}\right) \otimes\left(\beta X_{i j}^{(2)}+2 I_{d}\right) \\
& T_{i j}^{(3)}=\left(E_{i i}+\mathrm{i} E_{i j}-\mathrm{i} E_{j i}+E_{j j}\right) \otimes\left(\beta X_{i j}^{(3)}+2 I_{d}\right) \\
& T_{i j}^{(4)}=\left(E_{i i}-\mathrm{i} E_{i j}+\mathrm{i} E_{j i}+E_{j j}\right) \otimes\left(\beta X_{i j}^{(4)}+2 I_{d}\right) .
\end{aligned}
$$

For $1 \leq i \leq n$ we further introduce

$$
\tilde{T}_{i}=E_{i i} \otimes\left[-\beta \sum_{j \neq i}\left(X_{i j}^{(1)}+X_{i j}^{(2)}+X_{i j}^{(3)}+X_{i j}^{(4)}\right)+2 \beta X_{i i}^{(1)}-8(n-1) I_{d}\right],
$$

where we write, for convenience, $X_{j i}^{(\cdot)}=X_{i j}^{(\cdot)}$ for $i<j$. Let

$$
\tilde{Y}_{d}=\frac{1}{2 \beta \sqrt{n}}\left[\sum_{1 \leq i<j \leq n} \sum_{s=1}^{4} T_{i j}^{(s)}+\sum_{i=1}^{n} \tilde{T}_{i}\right] .
$$

A direct computations shows that $\tilde{Y}_{d}$ follows a GUE $n d$ distribution. Define

$$
Y_{d}:=x I_{n d}+\tilde{Y}_{d}=\frac{1}{2 \beta \sqrt{n}}\left[\sum_{1 \leq i<j \leq n} \sum_{s=1}^{4} T_{i j}^{(s)}+\sum_{i=1}^{n} T_{i}\right]
$$

where the new diagonal blocks read

$$
T_{i}=E_{i i} \otimes\left[-\beta \sum_{j \neq i}\left(X_{i j}^{(1)}+X_{i j}^{(2)}+X_{i j}^{(3)}+X_{i j}^{(4)}\right)+2 \beta X_{i i}^{(1)}-8(n-1) I_{d}+2 x \beta \sqrt{n} I_{d}\right] .
$$

We look now for the range of $x$ such that equation (7) is a separable decomposition for the matrix $Y_{d}$. Since $\beta<1$, each of the operators $\beta X_{i j}^{(1)}+2 I_{d}$ is, almost surely as $d \rightarrow \infty$, positive semidefinite; hence, the same hols for all $T_{i j}^{(\cdot)}$. Moreover, for all $i$, the limiting eigenvalue distribution of the random matrix $T_{i}$ is

$$
\mathrm{SC}_{2 x \beta \sqrt{n}-8(n-1), 2 \beta \sqrt{n}},
$$

which has positive support if and only if

$$
x \geq 2+\frac{4(n-1)}{\beta \sqrt{n}} .
$$

Optimizing over the values of $\beta \in(0,1)$, we conclude that $x I_{n d}+\mathrm{GUE}_{n d}$ is separable whenever

$$
x>2+\frac{4(n-1)}{\sqrt{n}} .
$$

For the matrix $Z_{d}$ in (4), we have now that

$$
Z_{d}=\alpha\left(\frac{2}{\alpha}+\mathrm{GUE}_{n d}\right)
$$


is separable whenever

$$
\frac{2}{\alpha}>2+\frac{4(n-1)}{\sqrt{n}}
$$

which finishes the proof.

Corollary 5.9. In almost all directions, for any $n>16$, the convex body of separable states is contained between two euclidean balls of relative radii $4(\sqrt{n}+2(n-1)) / n$. For $n$ large, the ratio that we can obtain is of the order of 8 .

Note that the comparison is quite interesting with [15, Corollary 3] that says that the largest euclidean ball inside separable sets in our above setup is of radius $1 / n$. Obviously our order of $1 / \sqrt{n}$ is much larger, however, our result is true 'only' in 'almost all' directions, so it is actually compatible - and of intrinsic interest.

Our results also complement the following finding of Aubrun, Szarek and Ye in [3], which can be stated informally as follows:

Theorem 5.10. Let $\rho \in \mathcal{M}_{n m}(\mathbb{C})$ be a random quantum state distributed along the induced measure with parameters $(n m, s)$. Then, there exist constants $c_{1,2}$ and a function $s_{0}=s_{0}(n, m)$ such that

$$
c_{1} n m \min (n, m) \leq s_{0} \leq c_{2} n m \log (n m)^{2} \min (n, m)
$$

and

(1) If $s<(1-\varepsilon) s_{0}$, the probability that $\rho$ is separable is exponentially low.

(2) If $s>(1+\varepsilon) s_{0}$, the probability that $\rho$ is entangled is exponentially low.

Remark 5.11. In the vein of the above theorem, it would be interesting to find good examples of typically l-separable non-centered GUE. Our construction above is specific to the one-separable case, and we do not see how to extend it in a non-trivial way to the l-separable setup.

5.3. Indecomposability. We recall [29, Definition 1.2.8] that a positive map $\Phi$ is called decomposable if it can be written as

$$
\Phi=\Phi_{1}+\top \circ \Phi_{2}
$$

where $T$ is the transposition map and $\Phi_{1,2}$ are completely positive maps.

Theorem 5.12. In the setting of Theorem 5.4, as soon as $2(2+\varepsilon)<\alpha \sqrt{n}$, almost surely as $d \rightarrow \infty$, the positive linear map $\Phi_{d}$ having Choi matrix $C_{d}$, is indecomposable.

Proof. This follows from the third point in Theorem 5.4: the map $\Phi_{d}$ detects (for $d$ large) the entanglement in the PPT state $Z_{d}$, hence it cannot be decomposable, see [29, Corollary 7.2 .5 and Proposition 7.3.6].

\section{REFERENCES}

[1] Anderson, G., Guionnet, A., and Zeitouni, O. An Introduction to Random Matrices. Cambridge University Press, 2009.

[2] Aubrun, G. Partial transposition of random states and non-centered semicircular distributions. Random Matrices: Theory Appl., 01, 1250001 (2012).

[3] Aubrun, G., Szarek, S. and Ye, D. Entanglement thresholds for random induced states. Comm. Pure and Appl. Math. 67 (1), pp. 129-171 (2014).

[4] Banica, T. and Nechita, I. Asymptotic eigenvalue distributions of block-transposed Wishart matrices. J. Theoret. Probab. 26 (2013), 855-869.

[5] Belinschi, S. T., Collins, B. and Nechita, I. Laws of large numbers for eigenvectors and eigenvalues associated to random subspaces in a tensor product. Inventiones Mathematicae, vol. 190, no. 3, 2012, pp. 647-697.

[6] Belinschi, S. T., Collins, B. and Nechita, I. Almost one bit violation for the additivity of the minimum output entropy. Preprint arXiv:1305.1567.0 
[7] Bercovici, H., and Voiculescu, D. Superconvergence to the central limit and failure of the Cramr theorem for free random variables. Probability Theory and Related Fields 103, no. 2 (1995): 215-222.

[8] Blackadar, B. Operator Algebras: Theory of $C^{*}$-Algebras and von Neumann Algebras. Springer-Verlag (2006).

[9] S. Braunstein, C. M. Caves, R. Jozsa, N. Linden and S. Popescu. Separability of very noisy mixed states and implications for NMR quantum computing. Physical Review Letters 83.5 (1999): 1054.

[10] Cerf, N.J., Adami C. and Gingrich, R.M. Reduction criterion for separability. Phys. Rev. A 60 (1999), 898-909.

[11] Collins, B. and Male, C. The strong asymptotic freeness of Haar and deterministic matrices Annales Scientifiques de l'École Normale Supérieure. Volume 47, fascicule 1 (2014), 147 - 163.

[12] B. Collins and I. Nechita. Random quantum channels II: Entanglement of random subspaces, Rényi entropy estimates and additivity problems. Advances in Mathematics 226.2 (2011): 1181-1201.

[13] M. D. Choi, Completely Positive Linear Maps on Complex Matrices, Lin. Alg. Appl, 10, 3, pp. 285-290 (1975).

[14] O. Fawzi, P. Hayden P. Sen. From low-distortion norm embeddings to explicit uncertainty relations and efficient information locking. Journal of the ACM (JACM) 60.6 (2013): 44.

[15] Gurvits, L., and Barnum, H. Largest separable balls around the maximally mixed bipartite quantum state. Phys. Rev. A 66, 062311 (2002).

[16] M. B. Hastings. Superadditivity of communication capacity using entangled inputs. Nature Physics 5.4 (2009): 255-257.

[17] P. Hayden, M. Horodecki, A. Winter and J. Yard. A decoupling approach to the quantum capacity. Open Systems \& Information Dynamics 15.01 (2008): 7-19.

[18] P. Hayden, D. Leung, P. Shor and A. Winter. Randomizing quantum states: Constructions and applications. Communications in Mathematical Physics 250.2 (2004): 371-391.

[19] P. Hayden and A. Winter. Counterexamples to the maximal p-norm multiplicativity conjecture for all $p_{\dot{\varepsilon}} 1$. Communications in mathematical physics 284.1 (2008): 263-280.

[20] Horodecki, M. and Horodecki, P. Reduction criterion of separability and limits for a class of distillation protocols. Phys. Rev. A 59 (1999), 4206-4216.

[21] Horodecki, M.,Oppenheim, J., and Winter, A. Quantum state merging and negative information. Communications in Mathematical Physics 269, no. 1 (2007): 107-136.

[22] Hou, J., Li, C.-K., Poon, Y.-T., Qi, X., and Sze, N.-S. Criteria and new classes of k-positive maps. arXiv:1211.0386.

[23] Kargin, V. On superconvergence of sums of free random variables. The Annals of Probability (2007): 1931-1949.

[24] Nica, A. and Speicher, R. Lectures on the combinatorics of free probability. Cambridge Univ. Press (2006).

[25] Peres, A. Separability criterion for density matrices. Phys. Rev. Lett., 77 (1996), 1413-1415.

[26] B. Terhal, and P. Horodecki, Schmidt number for density matrices, Phys. Rev. A 61, 040301(R) (2000).

[27] B. Schumacher and M. D. Westmoreland. Optimal signal ensembles. Physical Review A 63.2 (2001): 022308.

[28] G. Smith and J. A. Smolin. Extensive nonadditivity of privacy. Physical Review Letters 103.12 (2009): 120503.

[29] Størmer, E. Positive Linear Maps of Operator Algebras. Springer, 2013.

[30] W. F. Stinespring. Positive functions on $C$ *-algebras. Proceedings of the American Mathematical Society 6.2 (1955): 211-216.

[31] Voiculescu, D. Addition of certain non-commuting random variables. J. Funct. Anal. 66 (1986), 323-346.

[32] Voiculescu, D. Multiplication of certain noncommuting random variables. J. Operator Theory 18 (1987), 22232235.

[33] Voiculescu, D., Dykema, K. and Nica, A. Free random variables. Amer Mathematical Society, 1992.

Department of Mathematics, Graduate School of Science, Kyoto University, Kyoto 606-8502, Japan and Département de Mathématique et Statistique, Université d'Ottawa, 585 King Edward, Ottawa, ON, K1N6n5 Canada, CNRS, Institut Camille Jordan Université Lyon 1, 43 Bd du 11 Novembre 1918, 69622 Villeurbanne France

E-mail address: collins@math.kyoto-u.ac.jp

Physics Department, Stanford University. Varian Physics Bullding, 382 Via Pueblo Mall, Stanford, 94305-4060, USA.

E-mail address: phayden@stanford.edu

Zentrum Mathematik, M5, Technische Universität München, Boltzmannstrasse 3, 85748 Garching, Germany, and CNRS, Laboratoire de Physique Théorique, Toulouse, France

E-mail address: nechita@irsamc.ups-tlse.fr 